\title{
Minimally Invasive Approach for Treatment of Renal Calycenal Diverticulum
}

\author{
Ricardo Zerati , Marcos Tobias-Machado* \\ Marcos Tobias-Machado, Department of Urology, Hospitais da Rede D’Or, São Luis, São Paulo, Brasil
}

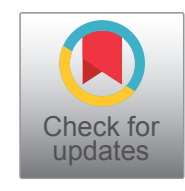

\begin{abstract}
The treatment of patients with renal intra-diverticular stones with minimal invasive surgery is always a challenge for the surgeon. We present here a report of a case of pure laparoscopic nephrolithotomy and another with a robotic approach for multiple calculi in renal calycenal diverticulum in two young female patients, previously treated by unsuccessful ureteroscopy in addition to two previous lumbotomies in one of the patients due to renal abscess.

In this unique and innovative method, pure laparoscopy or robotic assisted, allows a safe approach with high success rates. This technique avoided scarring from an open procedure and also extensive dissection of the ureter. This unique surgery can be considered as a confluence of endourology and laparoscopy, being another resource for the treatment of diverticular calculi by a minimally invasive approach.
\end{abstract}

\section{Introduction}

Although nephrolithiasis is quite common, the use of laparoscopic and robotic nephro-lithotomy is uncommon in this era of advanced invasive methods [1,2]. With technological advances, new robotic systems, 3D laparoscopy, energy sources, among others; have become the technique even more feasible since the treatment of patients with various stones in renal diverticula is always a challenge for the surgeon $[2,3]$. Here we present the report of two cases of nephrolithotomy, pure laparoscopic and robot assisted for intra diverticular renal calculi in anterior topography in two young women. This approach avoided open nephrolithotomy since there was no resolution with ureteroscopic approaches in both patients.

\section{Case presentation -1}

A 36-year-old girl sought emergency care due to fever, dysuria and low back pain. History of urinary tract infections from childhood with search for negative vesicoureteral reflux. The patient was submitted to hospitalization for clinical support and diagnostic clarification. The computed tomography (CT) was suggestive of renal calculi on the right, with size ranging from 5 to $8 \mathrm{~mm}$, diverticulum from the upper pole associated with a liquid collection (Figure 1).

Pyelography prior to hospitalization showed a calicinal diverticulum anterior to the right in addition to a small pyelic calculus (Figure 2). The DTPA scan showed a GFR of 45.5 $\mathrm{ml} / \mathrm{min}$ for the right kidney and $55.6 \mathrm{ml} / \mathrm{min}$ for the left kidney. As the patient was a young female, with a history of 2 lumbotomies due to renal abscess and ureteroscopy (URS) due to nephrolithiasis, we gave her the option of this unique method of laparoscopic nephrolithomy to avoid scar on the abdomen in open surgery and due to previous approaches unsuccessfully.

After the complete preoperative evaluation, resolution of the infectious condition and valid written consent, the patient received general anesthesia and the left lateral decubitus position with the right side up. The insertion of laparoscopic portals was done with caution due to previous surgeries.

The right upper renal pole was meticulously dissected. The resection of the anterior wall of the diverticulum was performed using a harmonic scalpel and purulent secretion and the calculi were identified (Figure 3). During the resection, there was no leakage of serum since a ureteral catheter was passed at the beginning of the procedure and kept under irrigation. The diverticulum was marsupialized to avoid recurrence. A hemostatic suture in the diverticular bed with Vicryl 2-0 and a drain was maintained. The ureteral catheter was removed at the end of the procedure. The patient was discharged on the third day without complaints.

*Corresponding author: Dr.Marcos Tobias-Machado, Department of Urology, Hospitais da Rede D’Or, São Luis, São Paulo, Brazil

Accepted: June 18, 2021

Published online: June 21, 2021

Citation: Zerati R, Tobias-Machado M (2021) Minimally Invasive Approach for Treatment of Renal Calycenal Diverticulum. Adv Laparoscopy 4(1):93-95 


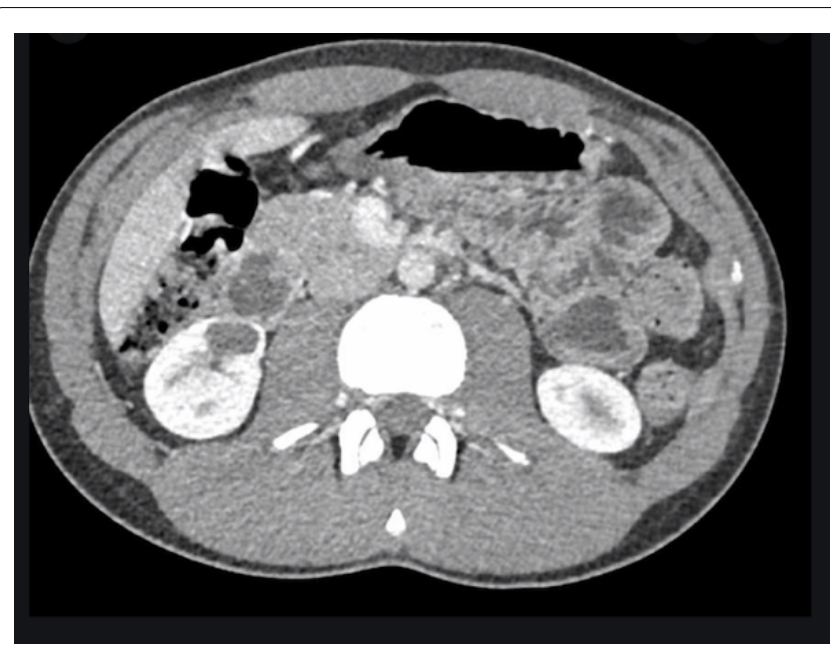

Figure 1: CT showing fluid collection in diverticulum area and calculi

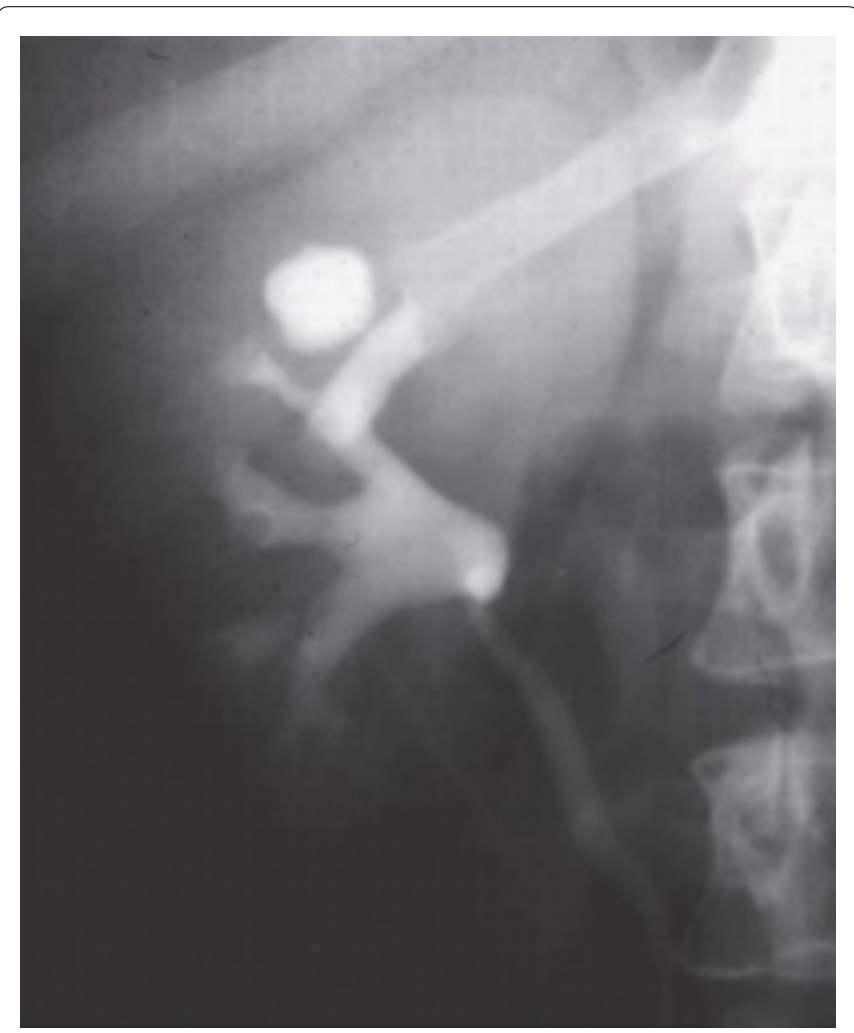

Figure 2: Previous pyelography showing calyceal diverticulum and a pyelic calculi

\section{Case presentation -2}

A 28-year-old girl came to our service due to sporadic low back pain and 3 episodes of urinary tract infection (UTI) in the last 8 months. She performed 5 extracorporeal shock wave lithotripsy (ESWL) and 2 URS in the last 3 years due to nephrolithiasis. CT (Figure 4) shows anterior diverticulum at the upper pole of the right kidney, with multiple stones inside up to $8 \mathrm{~mm}$ (Figure 5).

As the patient was young, and has had many previous unsuccessful surgeries, we gave her the option of this unique method of robotic nephrolithomy. After a complete preoperative evaluation including negative urine culture and valid written consent, the patient received general anesthesia and a left lateral decubitus position was performed.

The Gerotta's fascia was dissected to access the right kidney. Laparoscopic ultrasound was used for greater precision in the identification of calculations (Figure 6). After removing the calculi, the anterior wall of the diverticulum was resected, the diverticular bed was fulgurated and a hemostatic suture at the diverticular edge with 3-0 v loc suture thread.

As in the other case, during the resection, there was no extravasation of serum, as a ureteral catheter was passed at the beginning of the procedure and kept under irrigation. The ureteral catheter was removed at the end of the procedure. The patient was discharged on the day after without any complaints

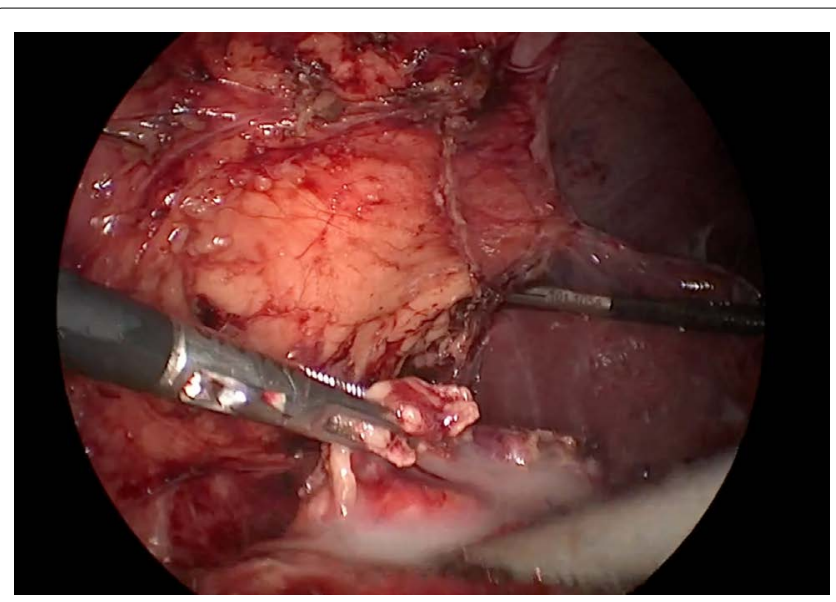

Figure 3: Diverticular opening with secretion and calculi output

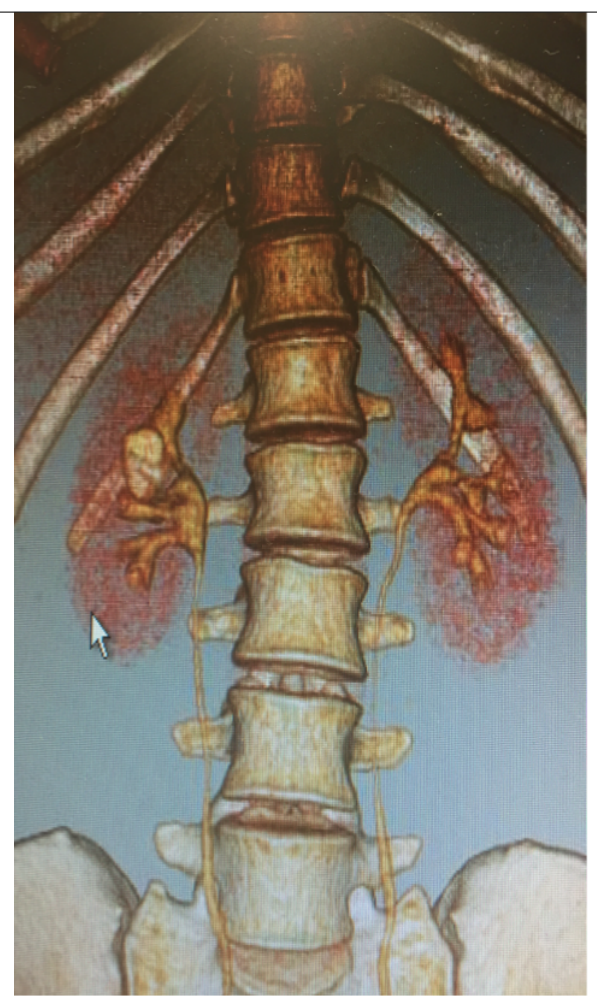

Figure 4: CT shows anterior diverticulum at the upper pole of the right kidney 


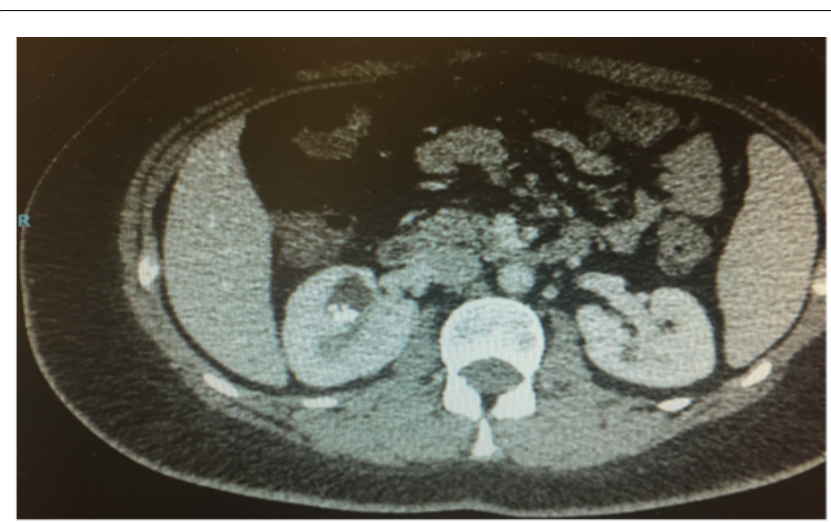

Figure 5: CT shows anterior diverticulum with multiple calculi

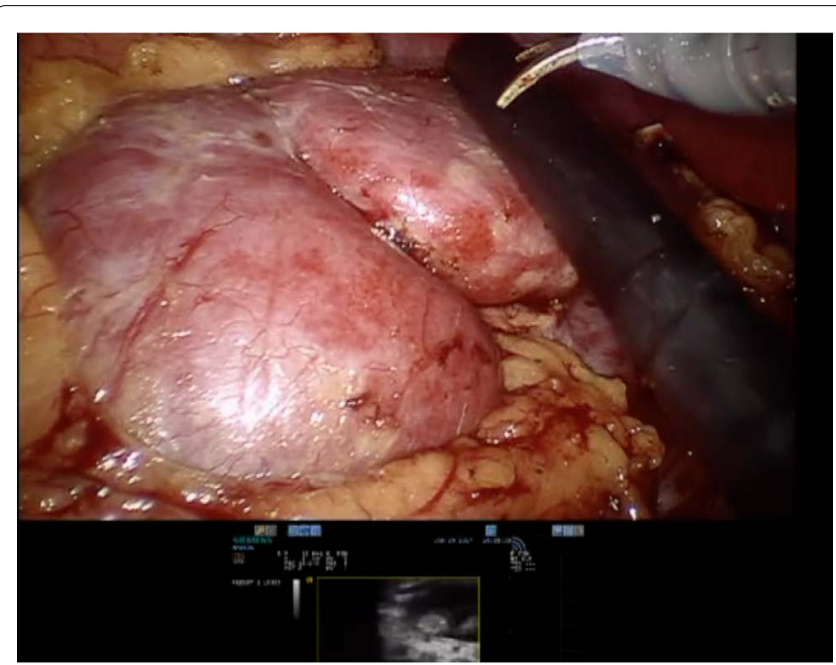

Figure 6: Use of laparoscopic ultrasound to better locate the stones, note images producing posterior acoustic shadow

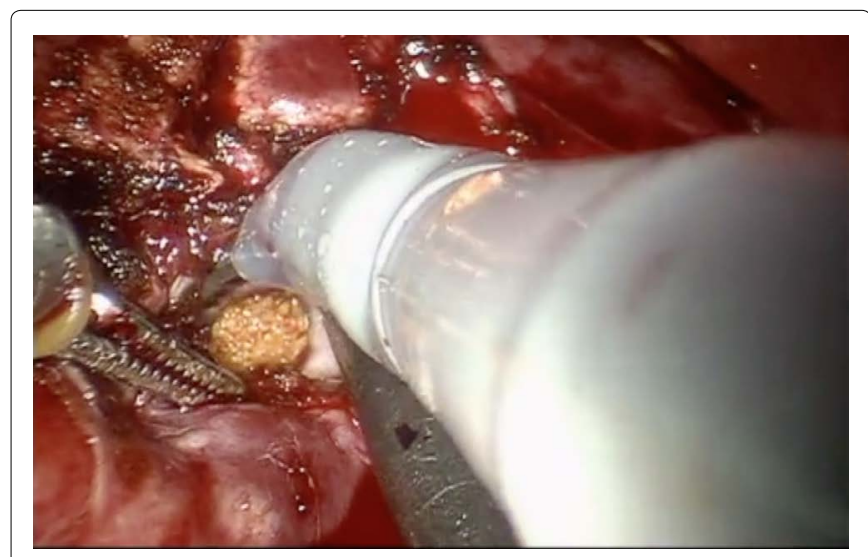

Figure 7: Robotic extraction of calculi and placement into a bag intra- abdominally

\section{Discussion}

Calyceal diverticula is a rare congenital urotheliumcoated, non-secreting cavities within the renal parenchyma that communicate with the collecting system through an infundibulum. Most are asymptomatic. When symptomatic, low back pain (30 - 40\%), calculations (10 - 50\%), repetition UTI / Pyelonephritis (20 - 25\%) are the main findings. [1,4] Diagnosed in $0.2-0.6 \%$ of patients with renal imaging and are more common in women.

Pure laparoscopy or assisted robotics are particularly useful for dealing with polar and anterior diverticulum injuries with a large stone load and a closed ostium, and this minimally invasive approach is well described in the literature, especially for the anterior ones not amenable for percutaneous approach [2].

The intraoperative visualization of the diverticulum is easy in people with polar location and thin parenchyma. If visual inspection does not reveal the diverticulum, the use of intraoperative ultrasound will aid in the accurate identification $[2,4]$.

Complications of minimally invasive surgery are related to abdominal or retroperitoneal access, pneumoperitoneum, tissue dissection, especially in patients with previous surgery or incisional hernia formation. No serious complications were reported in our review.

\section{Conclusion}

This is the case report that shows the use of minimally invasive surgery to treat multiple diverticular stones. The essence of this procedure is minimal surgical trauma to the kidney and ureter, the cosmetic benefit to the patient, shorter hospital stay and recovery, offering an excellent method of minimally invasive treatment, taking into account the nonresolution of the condition with other techniques used. Four months later, the patients are asymptomatic in outpatient follow-up.

\section{Refrences}

1. Smyth Niamh, Somani Bhaskar, Rai Bhavan, et al. (2019) Treatment Options for Calyceal Diverticula. Curr Urol Rep 23: 37.

2. Canales Benjamin , Monga Manoj (2003) Surgical management of the calyceal diverticulum. Current opinion in urology 13: 255260.

3. Waingankar Nikhil, Al-Hayek Samih, Smith Arthur, et al.(2014) Calyceal Diverticula: A comprehensive review Rev Urol 16: 2943.

4. Matlaga Brian, Miller Nicole, Terry Colin (2007) The pathogenesis of calyceal diverticular calculi. Urological research. 35: 35-40.

DOI: $10.36959 / 367 / 448$

Copyright: (C) 2021 Mohamed MN. This is an open-access article distributed under the terms of the Creative Commons Attribution License, which permits unrestricted use, distribution, and reproduction in any medium, provided the original author and source are credited. 